\title{
A Minimum of Two Years Results of Magnetically Controlled Growing Rods for Early Onset Scoliosis
}

\author{
Burstein $\mathbf{J}^{1,2}$, Rupprecht $\mathbf{M}^{1,2}$, Kunkel $\mathbf{P}^{3}$, Spiro $A \mathbf{S}^{1,2}$, Hagemann $\mathbf{C}^{3}$, Vettorazzi $E^{4}$, Stücker $\mathbf{R}^{1,2}$ and Ridderbusch $\mathbf{K}^{1,2^{*}}$ \\ ${ }^{1}$ Department of Pediatric Orthopaedics, Altona Children's Hospital, Hamburg, Germany \\ 2Department of Orthopaedics, University Medical Center Hamburg-Eppendorf, Germany \\ ${ }^{3}$ Department of Pediatric Neurosurgery, Altona Children's Hospital, Hamburg, Germany \\ ${ }^{4}$ Department of Medical Biometry and Epidemiology, University Medical Center Hamburg-Eppendorf, Hamburg, Germany
}

"Corresponding author: Ridderbusch K, Department of Pediatric Orthopaedics, Altona Children's Hospital, Hamburg, Germany, Tel: 00494088908382; E-mail: karsten.ridderbusch@kinderkrankenhaus.net

Rec Date: December 04, 2017; Acc Date: December 12, 2017; Pub Date: December 16, 2017

Copyright: (c) 2017 Burstein J, et al. This is an open-access article distributed under the terms of the Creative Commons Attribution License, which permits unrestricted use, distribution, and reproduction in any medium, provided the original author and source are credited.

\begin{abstract}
Objectives: To determine mid-term results and complications of a single center on magnetically controlled growing rod (MCGR) technique in Early Onset Scoliosis (EOS). Recently a number of preliminary reports about safety and effectiveness of MCGR have been published. We present a minimum of two years follow up of the MCGR treatment. All patients were treated in one center with the same protocol.
\end{abstract}

Methods: 22 patients with EOS of different etiologies underwent primary surgical treatment with MCGR in double rod technique. There were 15 female and 7 male patients. Average age at surgery was 8.8 (range 4.6-14.3). Mean follow-up was 31 (24-46) months. Cobb angle changes, T1-T12 and T1-S1 length were calculated. Results were analyzed to measure correction of spinal deformity and gain in spinal length.

Results: The mean preoperative curve measured $61^{\circ}(40-96)$ and improved to $28^{\circ}(11-53)(p<0.001)$ after surgery. At follow-up the Cobb angle was further reduced to $26^{\circ}(11-64)(p=0.54)$. The T1-T12- as well as the T1-S1length increased significantly $(p<0.001)$. The average preoperative thoracic kyphosis decreased from $44^{\circ}(-32-86)$ to $28^{\circ}(9-50)$ after surgery, respectively to $35^{\circ}(8-62)$ at time of FU. Four patients developed a junctional kyphosis. Three of them had to be revised. One patient developed a lumbar "adding on", one patient a deep wound infection and in another patient a screw pullout occurred that all required revision.

Conclusion: The study supports that the MCGR is an efficient and safe technique to treat EOS. Morbidity and complications are less than treatment with conventional GR's. After 2 years transcutaneous lengthening is still possible without loss of correction and autofusion has not yet been detected.

Keywords: Scoliosis; Non-fusion techniques; MCGR; Early onset scoliosis

\section{Introduction}

The treatment of Early Onset Scoliosis (EOS) has developed significantly over the last years. The main goal of any treatment is to maintain normal thoracic growth and pulmonary function and to correct the deformity without negatively affecting the patient quality of life. In young patients below age of 5 casting is an effective way to correct the deformity, halt progression and at least to buy some time and avoid early surgery. Over the age of 5 years casting is less effective and less well accepted $[1,2]$. In those patients surgery has to be considered especially for neuromuscular and congenital deformities because bracing those patients has been found to be ineffective $[2,3]$. As a consequence of progressive EOS deterioration of pulmonary function and the development of a thoracic insufficiency syndrome (TIS) may develop. To avoid restrictive lung disease a thoracic height (T1-T12) of at least $18-22 \mathrm{~cm}$ at the time of maturity should be achieved $[4,5]$. The aim of novel treatments is to allow the spine to grow while controlling the curve until the child is old enough for spinal fusion. But the need of repeated surgical interventions to lengthen the rod in conventional growing rods leads to increasing risk of infections and further complications [6,7]. Therefore, the technology of magnetically growing rods (MCGR) gains more and more acceptance and is increasingly applied. Lengthening can be achieved non-invasively avoiding multiple surgeries. MCGR have been reported to be safe and effective at short-term follow up [8-11]. The MCGR-technique combines the effectiveness of a dual rod procedure, but reduces morbidity and complication rates. However, mid-term studies on MCGR are rare. Teoh and Winson reports on 8 patients (3 single rod, 5 double rod technique) with a minimum on 44 months follow up [12]. This single center investigation reports on a minimum of two years results on safety and efficiency of MCGR adhering to a specific lengthening protocol every 4 months.

\section{Patients and Methods}

Approval was obtained by the ethical committee prior to the study. From June 2011 until June 2013, a total of 22 patients (15 girls and 7 boys) with EOS underwent the MCGR procedure as primary surgery at our institution. Inclusion criteria: (I) EOS of any etiology (Figure 1); (II) scoliosis with Cobb-angel $>40^{\circ}$, and/or annual progression $>5^{\circ}$, and/or failed non-operative treatment including physiotherapy and 
bracing; (III) a minimum follow-up of at least 24 months and (IV) at least 6 lengthening procedures after surgery. At time of surgery the average age was 8.8 (range 4.6 to 14.3 years). The $14+$ year old male patient had a skeletal bone age of $11+8$ years.
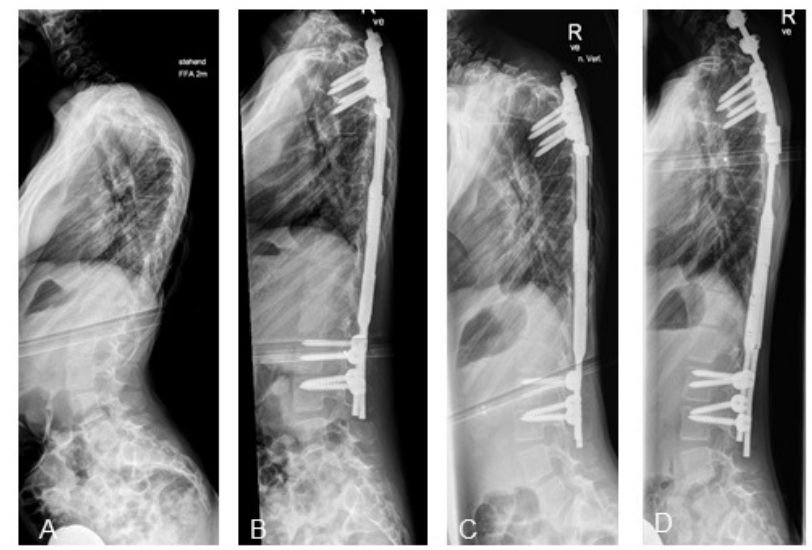

Figure 1: 11-year-old girl with thoracogenic scoliosis developing a junctional kyphosis 6 month after MCGR-implantation (A) preoperative view, (B) post-operative view, (C) junctional kyphosis 6 months postoperatively, (D) after revision.

In general, all idiopathic and in some extent syndromic and neuromuscular patients were braced before. We perform MCGR between the age of 4 and a minimum of 2 years of spine growth left.

\section{Surgical technique}

The MCGR consist of a single-use sterile titanium spinal distractible rod with an enlarged mid portion containing a magnetically drivable lengthening mechanism. Rotation of the internal magnet remotely induced by an External Remote Controller (ERC) causes the rod to be lengthened. Selection criteria for MCGR implantation do not differ from standard Growing Rod techniques [13]. All patients in this study were primary cases with 2nd generation MAGEC (including an additional keeper plate next to the internal magnet) who underwent surgery in double rod technique with general anaesthesia and endotracheal intubation. We applied all of our Growing Rod constructs in standard/offset technique. According to standard methods, two posterior midline approaches were made close to the most cephalad and caudal vertebral levels to anchor the rods. In general, only 1 motion segment of two adjacent vertebrae was instrumented with pedicle screws and fusion of these segments was performed to maintain implant stability and preserving sagittal balance. On grounds of dysplastic pedicles laminar hooks were used. In addition, sublaminar bands were inserted around the ribs belonging to the upper instrumented vertebra to avoid migration and ploughing of screws. We are using woven polyester bands for additional fixation, F.a. Zimmer Spine, 7375 Minneapolis, USA. Correction of the curve is obtained by distraction between the anchor points assisted by manual pressure like in the push prone technique.

\section{Lengthening procedure}

Lengthenings were performed every 4 months as outpatient procedures. After taking standing X-rays in two planes the patient was positioned prone. The hand-held External Remote Controller (ERC) was placed externally over the patient's back at the level of the internal rod. Distraction length between $3 \mathrm{~mm}$ to $5 \mathrm{~mm}$ according to Dimeglio growth charts [14] was planned with the concavity being distracted 1 $\mathrm{mm}$ more than the convexity. The amount of distraction was read from the controller screen. Post distraction radiographs PA were taken to confirm rod lengthening. The maximum amount of possible distraction of the standard $5.5 \mathrm{~mm}$ MCGR is $4.8 \mathrm{~cm}$. Achieving the maximum amount, the rod has to be changed surgically. Up to now, no MCGR achieved maximum amount of distraction with the need of revision. Recently we added the use of diagnostic ultrasound to measure the amount of lengthening during the distraction procedure to reduce the amount of post distraction X-rays.

\section{Radiographic analysis}

Before surgery standing $\mathrm{x}$-rays of the entire spine were obtained in 2 planes. In addition, concave and convex bending films were ordered. Before and after lengthening procedures standing X-rays in 2 planes were routinely obtained. All measurements were performed independently by two pediatric orthopedic surgeons. The measurements included the degree of deformity (Cobb angle), determination of kyphosis (T1-T12) and lordosis (L1-L5) and T1-T12 and T1-S1 heights. Discrepancies of the measurements were discussed until an agreement was found. The Intra-Class-Correlation coefficient (ICC) demonstrates a high rate of intra observer value between the two evaluators. The ICC for TH1-Th12 is 0.996, TH1-S1 0.999 and Cobb angle 0.989 .

\section{Statistics}

The data was analyzed using SPSS statistical package (version 20, SPSS Inc., Chicago, IL). Descriptive statistics were given as mean and range (minimum to maximum). To compare pre-and post-treatment data a student paired t-test was used. Statistical significance was assigned to $\mathrm{p}$ values $<0.001$. The intra observer value is measured by using the intra class correlation coefficient (ICC) 0-1.

\section{Results}

In total twenty-two patients were included in the study. 20 patients had a thoracic, 1 had a lumbar and 1 a thoracolumbar scoliosis. The aetiologies of EOS included 7 syndromic, 5 neuromuscular, 4 neurofibromatosis, 4 idiopathic, and 2 thoracogenic deformities. All patients had at least 6 distractions (mean 8 , range 6 to 11; Table 1) during an average time of FU of 31 months (24 to 46). Braces were not prescribed after surgery. Until now, none of the patients has reached skeletal maturity. Afterwards combined rod removal followed by dorsal fusion was planned.

\section{Radiographic findings}

The average preoperative Cobb-angle was $61^{\circ}$ (40 to 96) and improved to $28^{\circ}$ (11 to 53) after index procedure $(\mathrm{p}<0.001)$, representing a correction rate of $54 \%$. The average Cobb angle after the most recent lengthening was $26^{\circ}$ (11 to $\left.64 ; \mathrm{p}=0.54\right)$. The mean preoperative thoracic kyphosis (T1-T12) was $44^{\circ}$ (-32 to 86), and changed to $28^{\circ}$ ( 9 to 50$)$ after surgery $(\mathrm{p}<0.001)$, respectively to $35^{\circ}(8$ to 62$)$ at the most recent $\mathrm{FU}(\mathrm{p}<0.05)$. (Figure 2) Lumbar lordosis (L1L5) changed from $41^{\circ}\left(17\right.$ to $70^{\circ}$ ) before surgery to $31^{\circ}$ (7 to 54 , $\mathrm{p}<0.001)$ afterwards. 
Citation: Burstein J, Rupprecht M, Kunkel P, Spiro AS, Hagemann C, et al. (2017) A Minimum of Two Years Results of Magnetically Controlled

\begin{tabular}{|c|c|c|c|c|c|c|c|c|}
\hline Patient & Sex & $\begin{array}{l}\text { Age at OP } \\
\text { (months) }\end{array}$ & Diagnosis & Scoliosis & Primary curve & Time to FU (months) & $\begin{array}{l}\text { Lengthenings } \\
\text { (n) }\end{array}$ & Complication \\
\hline 1 & $\mathrm{f}$ & 70 & unknown muscular disease & neuromuscular & thoracic & 39 & 8 & none \\
\hline 2 & $\mathrm{f}$ & 55 & Prader-Willi-Syndrome & syndromic & thoracolumbar & 39 & 10 & none \\
\hline 3 & $\mathrm{f}$ & 92 & Loeys Dietz-Syndrome & syndromic & thoracic & 42 & 11 & $\begin{array}{l}\text { pull out, junctional } \\
\text { kyphosis }\end{array}$ \\
\hline 4 & $\mathrm{f}$ & 113 & spinal muskelatrophia typ III & neuromuscular & lumbar & 46 & 11 & none \\
\hline 5 & f & 74 & unknown muscular disease & neuromuscular & thoracic & 37 & 9 & none \\
\hline 6 & $\mathrm{~m}$ & 68 & Dandy-Walker-Syndrome & syndromic & thoracic & 37 & 8 & pull out \\
\hline 7 & $\mathrm{~m}$ & 99 & NF type I & $\mathrm{NF}$ & thoracic & 37 & 9 & none \\
\hline 8 & $\mathrm{f}$ & 91 & NF type I & NF & thoracic & 36 & 9 & none \\
\hline 9 & $\mathrm{f}$ & 102 & Nemalin-myopathy & neuromuscular & thoracic & 29 & 7 & none \\
\hline 10 & $\mathrm{f}$ & 145 & rib fusion & thoracogenic & thoracic & 29 & 7 & junctional kyphosis \\
\hline 11 & $\mathrm{f}$ & 110 & infantile cerebral palsy & neuromuscular & lumbar & 28 & 7 & none \\
\hline 12 & $f$ & 117 & Prader-Willi-Syndrome & syndromic & thoracic & 28 & 7 & junctional kyphosis \\
\hline 13 & $\mathrm{~m}$ & 105 & Hamartosis & thoracogenic & thoracic & 28 & 7 & none \\
\hline 14 & $\mathrm{f}$ & 126 & infantile & idiopathic & thoracic & 28 & 6 & none \\
\hline 15 & $f$ & 123 & infantile Marfan Syndrome & syndromic & thoracic & 27 & 7 & none \\
\hline 16 & $\mathrm{~m}$ & 172 & infantile & idiopathic & thoracic & 27 & 7 & none \\
\hline 17 & $\mathrm{f}$ & 130 & infantile & idiopathic & thoracic & 26 & 5 & none \\
\hline 18 & $\mathrm{~m}$ & 71 & NF type I & $\mathrm{NF}$ & thoracic & 25 & 5 & late infection \\
\hline 19 & $\mathrm{f}$ & 97 & NF type I & NF & thoracic & 25 & 6 & none \\
\hline 20 & $\mathrm{f}$ & 127 & Chromosometranslocation & syndromic & thoracic & 24 & 6 & adding on \\
\hline 21 & $\mathrm{~m}$ & 80 & infantile & idiopathic & thoracic & 24 & 6 & none \\
\hline 22 & $\mathrm{~m}$ & 154 & Di Georg Syndrome & syndromic & thoracic & 24 & 6 & junctional kyphosis \\
\hline mean & $15 \mathrm{f} / 7 \mathrm{~m}$ & 106 & - & - & - & 31 & 8 & - \\
\hline \multicolumn{2}{|l|}{ SD } & 30 & - & - & - & 7 & 2 & - \\
\hline
\end{tabular}

Table 1: Demographic data and complications.

\begin{tabular}{|l|l|l|l|l|l|}
\hline Variables & Pre-op & Post-op & Change (\%) & FU & Change (\%) \\
\hline Cobb $\left(^{\circ}\right)$ & $61 \pm 14$ & $28 \pm 19$ & 54 & $26 \pm 13$ & 57 \\
\hline Kyphose $\left(^{\circ}\right)$ & $44 \pm 24$ & $28 \pm 12$ & 36 & $35 \pm 14$ & 21 \\
\hline Lordose $\left(^{\circ}\right)$ & $41 \pm 15$ & $31 \pm 12$ & 24 & $41 \pm 9$ & 0 \\
\hline T1-T12 $(\mathrm{cm})$ & $18.3 \pm 2.5$ & $20.4 \pm 2.6$ & 12 & $22.5 \pm 2.7$ & 23 \\
\hline T1-S1 $(\mathrm{cm})$ & $29.6 \pm 4.3$ & $33.1 \pm 4.3$ & 12 & $35.9 \pm 3.9$ & 21 \\
\hline
\end{tabular}

$20.4 \mathrm{~cm}$ (15.2 to 25.9$)$ postoperatively $(\mathrm{p}<0.001)$, and to $22.5 \mathrm{~cm}(17.4$ to 28.2) at $\mathrm{FU}(\mathrm{p}<0.001)$. The average increase of T1-T12 length after index procedure until final $\mathrm{FU}$ was $0.7 \mathrm{~mm}$ per month. The average T1-S1 height increased from $29.6 \mathrm{~cm}$ (21.7 to 37.7$)$ to 33.1 (25.9 to 40.8) postoperatively $(\mathrm{p}<0.001)$ to $35.9 \mathrm{~cm}(28.6$ to 44.4$)$ at $\mathrm{FU}$ $(\mathrm{p}<0.001)$. The average gain of length after the index procedure until FU was $1 \mathrm{~mm}$ per month. Radiographic measurements are listed in Table 2.

\section{Complications}

Table 2: Radiographic analysis.

At the time of $\mathrm{FU}$ the average lordosis increased to $41^{\circ}$ (26 to 58 , $\mathrm{p}=0.84)$. The T1-T12 length increased from $18.3 \mathrm{~cm}(13.1$ to 23.4$)$ to

Neither intraoperative nor neurologic complications nor rod fractures were observed. Loss of distractions after lengthening which were seen in some patients with first generation rods were not seen with second generation rods. Four patients developed a junctional 
kyphosis, three of them had to be revised. All of them were relatively rigid and had a preoperative kyphosis of more then $50^{\circ}$. One patient had a migration of screws, one a late deep infection and one a lumbar adding on which was resolved by revision and inclusion and instrumenting two more additional lumbar levels.
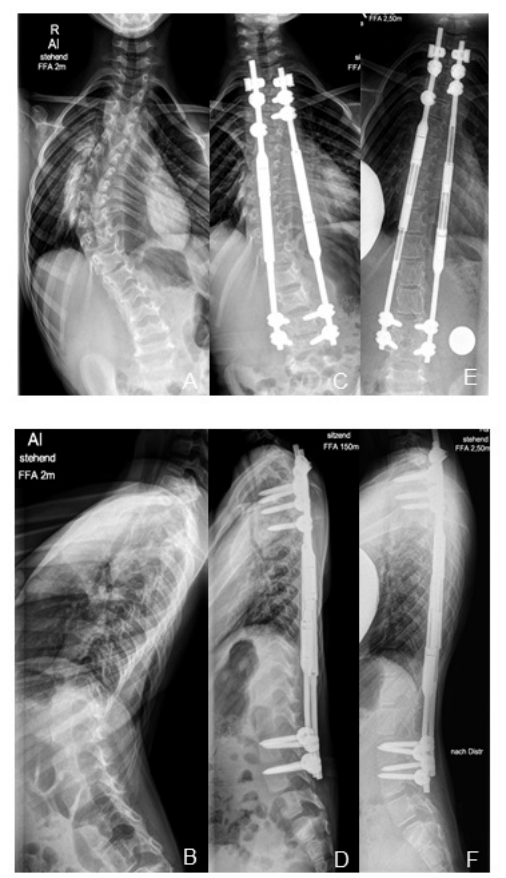

Figure 2: 6-year-old girl with an undefined muscle disease (A-B) preoperative view, (C-D) after MCGR-implantation, (E-F) 42 months follow up.

\section{Discussion}

The progression of spinal deformity in EOS requires early treatment and close observation. Different etiologies, comorbidities and the broad variety of treatment modalities make the management of EOS challenging $[2,3,15-19]$. Bracing is especially in neuromuscular and syndromic patients often not accepted or less effective. Fusion techniques result in spinal fusion limiting the growth of the thoracic spine and thoracic cage. Therefore, there is insufficient space for pulmonary alveolar growth leading to restrictive lung disease [20]. Karol and Johnston reported that the amount of fused segments significantly correlates with vital capacity [4]. Therefore, the idea and application of a non-fusion technique allowing natural spine and chest growth is attractive. The aim is to reach at least $22.5 \mathrm{~cm}$ of T1-T12 length to reduce the risk from developing a restricted lung disease. The most commonly used is the dual growing rod technique [13,21-24]. However, repeated surgical interventions required to lengthen the rods are associated with a high complication rate and possible psychological dysfunction [25]. Treatment with MCGR avoids repeated surgery and offers correction of deformity and maintaining growth with much less associated morbidity [8-10]. For children between age 5-10 years a normal spine growth T1-S1 with rates of $11 \mathrm{~mm} /$ year can be expected [14]. In this study T1-T12 length increased by $7 \mathrm{~mm} /$ year, the T1-S1 length by $12 \mathrm{~mm} /$ year, representing regular growth rates. The achieved distraction length is also comparable to standard GR. Akbarnia and
Marks described for standard dual growing rod technique of progressive early onset scoliosis an average T1-S1 length increase of $12,1 \mathrm{~mm}$ per year [13]. The best lengthening protocol in order to achieve the best results in terms of correction and avoiding complications is still to be established. It is controversial if application of maximum force during distraction or more gentle distraction and guiding growth according to Dimeglio data is preferable. In addition, the frequency of lengthenings is debated. Recommendations range from once per month to every 6 months. For conventional Growing Rods Akbarnia and Breakwell demonstrated that patients undergoing more frequent lengthenings than every 6 months had significantly greater spinal growth and better curve correction compared to those who underwent rod lengthenings at more than 6 months intervals [21]. In principle the best would be to lengthen the rod every single day to support regular growth. The decision to perform lengthenings every 4 months was based on the fact that many patients live far away from our institution and logistic reasons. Another reason was to perform lengthenings more frequently than with conventional growing rods but on the other hand trying to reduce the amount of necessary X-rays and radiation for the children. Our goal is to maintain normal spinal growth according to the Dimeglio growth charts and not to apply maximum forces during distraction. After index surgery we gain a correction of the cobb angle in the coronal plane from $61^{\circ}$ to $28^{\circ}$ $(\mathrm{p}<0.001)$. In the following lengthenings up to final follow up we achieved a slightly correction in average to $26^{\circ}$ but this was no statistical significant $(p=0.54)$. Therefore, we had to determine that after initial correction of the curve in the coronal plane we could achieve in most patients only a stabilization of the curve. These results are comparable to Akbarnia and Hickey $[8,11]$. We try to gain further correction during our lengthening procedure by applying more length on the concave rod.

Our experience highlights that there is a difference between planned amount of distraction as read from the screen of the ERC and amount of distraction as read from radiographs. It seems that true distraction is only $50 \%$ of intended distraction. Rolton and Thakar found in their case series of MCGR an average of only $35 \%$ of length gained after distraction compared to the planned length [26]. The reason for this is still unclear and has to be investigated in further studies. A combination of beginning autofusion and loss of applied distraction force during lengthening might be the cause. This has to be taken in account when planning distractions. Based on these results we decided to perform the distraction every 4 month with the aim to distract the spine more quickly than the predicted spinal growth rate to compensate the loss of true distraction and to achieve a slightly better curve correction. More frequently distractions are theoretically superior. However, this is very difficult to pursue in a busy clinical practice. Ultrasound measurements have been shown to be a good alternative to radiographs obtained after distractions. However, loosening of anchor points, ploughing of screws or rod fractures can only be detected on radiographs. Recently published results showing equivalent measurements with ultrasound and radiographs $[27,28]$. In our institution we have gained ample experience with ultrasound but have not established it as a routine to substitute radiography. As already discussed there are a number of complications in EOS treatment with conventional growing rod techniques not only due to repeated surgical procedures but also due to the type of implants and its position as well as the patient's age at initial treatment $[6,7]$. Common complications are deep wound infections, implant failure or breakage, prominent hardware, proximal junctional kyphosis, crankshaft phenomenon, as well as auto fusion [8,22,29]. Each 
additional surgical procedure during treatment increases the risk of a complication by $24 \%$ [7]. Using the MCGR technique we did not observe any major perioperative complications in our case series. All of the patients were treated with 2 nd generation GR and loss of distraction as seen in first generation rods has no longer been encountered $[8,10]$. Breakage of actuator pins has not been seen in this case series, but was observed in 3 of our revision cases which were not part of this study. The development of proximal junctional kyphosis $(\mathrm{PJK})$ is still an issue related to non-fusion techniques. After initial reduction of the main thoracic kyphosis four patients in our series developed a junctional kyphosis after several lengthening. Three cases needed revision and were subsequently managed by surgery with Ponte osteotomies and instrumentation including the next superior level. All of them had thoracic kyphosis of more then $50^{\circ}$ before surgery.

\section{Conclusion}

It is our impression that reducing thoracic kyphosis significantly in those patients with existing hyperkyphosis may lead to PJK. We now accept preexisting kyphosis and bent more kyphosis into the upper thoracic region. With that approach no more PJK has been observed in our more recent cases. In one patient with Dandy Walker syndrome a loosening of the proximal fixation on the convex site occurred 2.5 years after MAGEC implantation which led to revision of the proximal fixation. We only used double rod $5.5 \mathrm{~mm}$ constructs and did not see any rod breakages yet. Rod breakage is more common in single rod constructs and with the use of $4.5 \mathrm{~mm}$ constructs Yang and Sponseller reported 86 rod fractures in 49 patients in a prospectively collected conventional growing rod database of 327 patients [30]. Until now breaks of MCGR are rare. Dannawi and Altaf reported on rod breakage in 2 of 34 cases treated with MCGR [10]. However, the use of $5.5 \mathrm{~mm}$ constructs probably adds more rigidity to the spine. Maintaining some amount of flexibility may be desirable. Therefore, usage of 5,0 rods may be a good compromise between flexibility and stability. One of the most significant problems with the use of conventional growing rods is the development of autofusion. Such spontaneous fusion of the instrumented segments might result from repeated surgical trauma, from forceful distractions, from unloading of tissues or the presence of these implants itself sitting intramuscular next to the spine. Until now all implants still lengthen so that we assume that a complete autofusion has not been occurred in our cases even after more than 4 years followup yet. But this presumption can only be proven until finally fusion is performed. Longer follow-up is needed to state that MCGR can reduce autofusion. The weakness of the study is its retrospective nature and the small number of patients included. However, the strength of this report is that all patients were treated by the same surgical procedure and the same distraction protocol which makes it easily comparable when other surgical procedures or protocols are evaluated. The MAGEC magnetic growing rod system is a safe and effective treatment for progressive EOS. In addition to an effective curve correction, it allows for a continuous spinal growth, which is similar to those of normal children. Multiple surgeries that were required with conventional technique can be avoided by non-invasive distraction that can be performed as an outpatient procedure. Consequently, the complication rate and morbidity has dropped significantly leading to better patient and parent satisfaction.

\section{References}

1. Mehta MH (2005) Growth as a corrective force in the early treatment of progressive infantile scoliosis. J Bone Joint Surg 87: 1237-1247.
2. Sponseller PD, Yazici M, Demetracopoulos C, Emans JB (2007) Evidence basis for management of spine and chest wall deformities in children. Spine 32: 81-90.

3. McMaster MJ, Macnicol MF (1979) The management of progressive infantile idiopathic scoliosis. J Bone Joint Surg 61: 36-42.

4. Karol LA, Johnston, C, Mladenov, K, Schochet P, Walters P, et al. (2008) Pulmonary function following early thoracic fusion in nonneuromuscular scoliosis. J Bone Joint Surg 90: 1272-1281.

5. Karol LA (2011) Early definitive spinal fusion in young children. Clin Orthop Relat Res 469: 1323-1329.

6. Sankar WN, Acevedo DC, Skaggs DL (2010) Comparison of complications among growing spinal implants. Spine 35: 2091-2096.

7. Bess S, Akbarnia BA, Thompson GH, Sponseller PD, Shah SA, et al. (2010) Complications of growing-rod treatment for early-onset scoliosis: Analysis of one hundred and forty patients. J Bone Joint Surg 92: 2533-2543.

8. Akbarnia BA, Cheung K, Noordeen H, Elsebaie H, Yazici M, et al. (2013) Next generation of growth-sparing technique: Preliminary clinical results of a magnetically controlled growing rod (mcgr) in 14 patients with early onset scoliosis. Spine 38: 665-670.

9. Cheung KM, Cheung JP, Samartzis D, Mak KC, Wong YW, et al. (2012) Magnetically controlled growing rods for severe spinal curvature in young children: A prospective case series. Lancet 379: 1967-1974.

10. Dannawi Z, Altaf F, Harshavardhana NS, El Sebaie H, Noordeen H (2013) Early results of a remotely operated magnetic growth rod in early-onset scoliosis. Bone Joint J 95: 75-80.

11. Hickey BA, Towriss C, Baxter G, Yasso S, James S, et al. (2014) Early experience of MAGEC magnetic growing rods in the treatment of early onset scoliosis. Eur Spine J 23: 61-65.

12. Teoh KH, Winson DM, James SH, Jones A, Howes J, et al. (2016) Magnetic controlled growing rods for early-onset scoliosis: A 4-year follow-up. Spine J 16: 34-39.

13. Akbarnia BA, Marks, DS, Boachie-Adjei O, Thompson AG, Asher MA (2005) Dual growing rod technique for the treatment of progressive earlyonset scoliosis: A multicenter study. Spine 30: 46-57.

14. Dimeglio A, Canavese F, Charles YP (2011) Growth and adolescent idiopathic scoliosis: When and how much? J Pediatr Orthop 31: 28-36.

15. Campbell RM Jr, Smith MD, Mayes TC, Mangos JA, Willey-Courand DB, et al. (2003) The characteristics of thoracic insufficiency syndrome associated with fused ribs and congenital scoliosis. J Bone Joint Surg 85: 399-408.

16. Campbell RM Jr, Smith MD (2007) Thoracic in sufficiency syndrome and exotic scoliosis. J Bone Joint Surg 89: 108-122.

17. Fernandes P, Weinstein SL (2007) Natural history of early onset scoliosis. J Bone Joint Surg Am 89: 21-33.

18. Goldberg CJ, Gillic I, Connaughton O, Moore DP, Fogarty EE, et al. (2003) Respiratory function and cosmesis at maturity in infantile-onset scoliosis. Spine 28: 2397-2406.

19. Pehrsson K, Larsson S, Oden A, Nachemson A (1992) Long-term followup of patients with untreated scoliosis. A study of mortality, causes of death, and symptoms. Spine 17: 1091-1096.

20. Burri PH (2006) Structural aspects of postnatal lung development alveolar formation and growth. Biol Neonate 89: 313-322.

21. Akbarnia BA, Breakwell LM, Marks DS, McCarthy RE, Thompson AG, et al. (2008) Growing spine study group. Dual growing rod technique followed for three to eleven years until final fusion: The effect of frequency of lengthening. Spine 33: 984-990.

22. Elsebaie HB, Yazici M, Thompson GH, Emans JB, Skaggs DL, et al. (2011) Safety and efficacy of growing rod technique for pediatric congenital spinal deformities. J Pediatr Orthop 31: 1-5.

23. Thompson GH, Akbarnia BA, Kostial P, Connie PK, Douglas GA, et al. (2005) Comparison of single and dual growing rod techniques followed through definitive surgery: A preliminary study. Spine 30: 2039-2044.

24. Thompson GH, Akbarnia BA, Campbell RM Jr (2007) Growing rod techniques in early-onset scoliosis. J Pediatr Orthop 27: 354-361. 
Citation: Burstein J, Rupprecht M, Kunkel P, Spiro AS, Hagemann C, et al. (2017) A Minimum of Two Years Results of Magnetically Controlled Growing Rods for Early Onset Scoliosis. J Spine 6: 401. doi:10.4172/2165-7939.1000401

Page 6 of 6

25. Flynn JM, Matsumoto H, Torres F, Ramirez N, Vitale MG (2012) Psychological dysfunction in children who require repetitive surgery for early onset scoliosis. J Pediatr Orthop 32: 594-599.

26. Rolton D, Thakar C, Wilson-MacDonald J, Nnadi C (2016) Radiological and clinical assessment of the distraction achieved with remotely expandable growing rods in early onset scoliosis. Eur Spine J 25: 3371-3376.

27. Stokes OM, O'Donovan EJ, Bow CH (2014) Reducing radiation exposure in early-onset scoliosis surgery patients: novel use of ultrasonography to measure lengthening in magnetically- controlled growing rods. Spine 39 2397-2404
28. Yoon WW, Chang AC, Tyler P, Butt S, Raniga S, et al. (2015) The use of ultrasound in comparison to radiography in magnetically controlled growth rod lengthening measurement: a prospective study. Eur Spine J 24: 1422-1426.

29. Farooq N, Garrido E, Altaf F, Noordeen H (2010) Minimizing complications with single submuscular growing rods: A review of technique and results on 88 patients with minimum two-year follow-up. Spine 25: 2252-2258.

30. Yang JS, Sponseller PD, Thompson GH, Behrooz AA, John BE, et al. (2011) Growing rod fractures: Risk factors and opportunities for prevention. Spine 36: 1639-1644. 